\title{
Clinical Factors Associated with Renal Outcome After Heart Transplantation
}

\author{
Junseok Jeon, ${ }^{1}$ MD, Hee Jin Kwon, ${ }^{1}$ MD, Heejin Yoo, ${ }^{2}$ MS, Darae Kim, ${ }^{3}$ MD, Yang Hyun Cho, ${ }^{4}$ MD, \\ Jin-Oh Choi, ${ }^{3}$ MD, Kyunga Kim, ${ }^{2}$ MD, Kiick Sung, ${ }^{4}$ MD and Hye Ryoun Jang, ${ }^{1}$ MD
}

\begin{abstract}
Summary
Cardiorenal syndrome (CRS) frequently occurs in end-stage heart failure patients waiting for heart transplantation (HT). Decision-making regarding simultaneous heart and kidney transplantation is an unresolved issue in these patients. We investigated clinical factors associated with renal outcome after HT. A total of 180 patients who received HT from 1996 to 2015 were included. Factors associated with early post-HT chronic kidney disease (CKD, estimated glomerular filtration rate $[\mathrm{eGFR}]<60 \mathrm{~mL} /$ minute $/ 1.73 \mathrm{~m}^{2}$ within 1 year post-HT), post-HT end-stage kidney disease (ESKD), and significant renal function improvement $(\% \Delta \mathrm{eGFR}>15 \%)$ at 1 year post-HT were analyzed. Early post-HT CKD and post-HT ESKD developed in 61 (33.9\%) and 8 (4.4\%) of 180 patients, respectively. Old age was only independently associated with early post-HT CKD and preexisting CKD tended to be associated with early post-HT CKD. Old age and preexisting CKD were independently associated with post-HT ESKD. Low pre-HT eGFR and preoperative renal replacement therapy were not associated with early post-HT CKD or post-HT ESKD. Young age, low pre-HT eGFR, and high \% $\%$ eGFR 1 month postHT were independently associated with significant renal function improvement. Preoperative renal function, including preoperative RRT, was not associated with post-HT mortality. In conclusion, preexisting CKD may impact renal outcomes after HT, but preoperative severe renal dysfunction, even that severe enough to require RRT, may not be a contraindication for HT alone. Our data suggest the necessity of early HT in end-stage heart failure patients with CRS and the importance of careful management during the early postoperative period.
\end{abstract}

Key words: Cardiorenal syndrome, End-stage heart failure

(Int Heart J 2021; 62: 850-857)

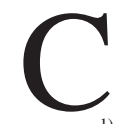
ardiorenal syndrome refers to conditions in which dysfunction of either the heart or the kidney leads to, or accelerates, dysfunction of the other organ. ${ }^{1)}$ In advanced heart failure patients, renal dysfunction is prevalent and associated with poor survival. ${ }^{2,3)}$ Heart transplantation (HT) is the definitive treatment for end-stage heart failure and it has shown good long-term survival. ${ }^{4,5}$ However, approximately $15-25 \%$ of patients waiting for HT have renal dysfunction, ${ }^{6,7)}$ which is associated with increased early and long-term mortality after HT. ${ }^{8.9)}$ The International Society for Heart and Lung Transplantation recommended simultaneous heart-kidney transplantation (HKT) for irreversible severe renal dysfunction (GFR $<30 \mathrm{~mL} /$ minute $\left./ 1.73 \mathrm{~m}^{2}\right){ }^{10)}$ However, practical difficulties such as the shortage of organs and lack of consensus on the criteria for irreversibility of renal dysfunction arise when considering HKT in all patients with eGFR $<30 \mathrm{~mL} /$ minute $/ 1.73 \mathrm{~m}^{2}$ waiting for HT.

The deterioration of renal function caused by hypop- erfusion or congestion in heart failure patients can be reversed before permanent renal damage occurs even if renal dysfunction is severe enough to require renal replacement therapy (RRT). ${ }^{11)}$ Predicting the reversibility of renal dysfunction in patients with heart failure is difficult and kidney biopsy is frequently infeasible due to patient condition or antiplatelet/anticoagulation therapy. Therefore, identifying non-invasively measurable clinical factors associated with renal outcome after HT is a research priority, but few studies have investigated factors associated with renal outcome after HT.

In this study, we investigated clinically important factors associated with renal outcome after HT in end-stage HF patients, focusing on changes in renal function and chronic kidney disease (CKD) prevalence.

\section{Methods}

Study cohort and data collection: We conducted a retro-

From the ${ }^{1}$ Division of Nephrology, Department of Medicine, Samsung Medical Center, Sungkyunkwan University School of Medicine, Seoul, Republic of Korea, ${ }^{2}$ Statistics and Data Center, Samsung Medical Center, Research Institute for Future Medicine, Seoul, Republic of Korea, ${ }^{3}$ Department of Cardiology, Samsung Medical Center, Sungkyunkwan University School of Medicine, Seoul, Republic of Korea and ${ }^{4}$ Department of Thoracic and Cardiovascular Surgery, Samsung Medical Center, Sungkyunkwan University School of Medicine, Seoul, Republic of Korea.

Address for correspondence: Hye Ryoun Jang, MD, Samsung Medical Center, Samsung Biomedical Research Institute, Sungkyunkwan University School of Medicine, 81 Irwon-ro, Gangnam-gu, Seoul 06351, Republic of Korea. E-mail: shinehr@skku.edu

Received for publication December 6, 2020. Revised and accepted February 15, 2021.

Released in advance online on J-STAGE July 17, 2021.

doi: 10.1536/ihj.20-775

All rights reserved by the International Heart Journal Association. 
spective cohort study of patients (age $\geq 15$ years) who underwent HT at Samsung Medical Center between January 1996 and December 2015. All patients included in the analyses were followed for at least 3 years after HT or death. Baseline demographic, clinical and laboratory parameters were extracted from electronic medical records. Data related to preoperative appliance and duration of any mechanical circulatory support including intra-aortic balloon pump, extracorporeal membrane oxygenation, and ventricular assist devices were collected. Patient outcomes including mortality and occurrence of postoperative adverse events such as prolonged mechanical ventilation for more than 3 days were also collected. After HT, all patients received immunosuppressive treatment including prednisolone, calcineurin inhibitors (CNIs), and purine inhibitors (azathioprine or mycophenolate mofetil) according to the center's routine protocol. Left ventricular ejection fraction was assessed by Simpson's biplane method according to the guidelines.

Evaluation of renal function and definitions of preexisting CKD/perioperative RRT: The modified Modification of Diet in Renal Disease (MDRD) formula was used to calculate estimated glomerular filtration rate (eGFR). ${ }^{12)}$ Preoperative baseline eGFR was defined as the median eGFR within 7 days before HT, and the preoperative baseline eGFR of patients who had already been receiving RRT before HT was considered to be $10 \mathrm{~mL} /$ minute $/ 1.73$ $\mathrm{m}^{2}$. Creatinine clearance $(\mathrm{CCr})$ was measured using 24hour urine collection. Proteinuria was measured using a standard urinary dipstick within 3 months before HT and semi-quantified as negative or trace (proteinuria $<30 \mathrm{mg}$ / $\mathrm{dL})$ and $\geq 1+(\geq 30$ to $100 \mathrm{mg} / \mathrm{dL})$ under normal range specific gravity. Pre-HT kidney ultrasound (US) scores were calculated as the sum of these scores as follows ${ }^{13)}$ : normal (0), increased parenchymal echogenicity (1), loss of corticomedullary differentiation (2), cortical thinning (3), and kidney atrophy (4). Preexisting CKD was defined as eGFR $<60 \mathrm{~mL} /$ minute $/ 1.73 \mathrm{~m}^{2}$ for at least 3 months before HT. ${ }^{14)}$ We defined the preoperative RRT group as patients who started RRT within 1 month before HT.

Outcomes: The primary endpoint was early post-HT CKD between 3 months and 1 year after HT, which was defined as eGFR $<60 \mathrm{~mL} /$ minute $/ 1.73 \mathrm{~m}^{2}$ for 3 consecutive months. Secondary outcomes were post-HT ESKD, eGFR after HT (post-HT eGFR), and significant improvement of renal function at 1 year after HT. Changes in renal function were assessed as percent change in eGFR (\% $\%$ eGFR) calculated as follows: $100 \times[$ (post-HT eGFR) - (pre-HT eGFR)]/pre-HT eGFR. Significant improvement of renal function was defined as $\% \Delta \mathrm{eGFR}>15 \%$ at 1 year after HT. ${ }^{15)}$ Subgroup analyses were performed according to pre-HT renal function (pre-HT eGFR and preoperative RRT).

Statistical analysis: Continuous variables are expressed as the mean \pm standard deviation (SD) or median (interquartile range, IQR), and categorical variables as the number (percentage, \%). Comparisons between groups were performed using Student's t-test or the Mann-Whitney test for continuous variables, and the chi-square test or Fisher's exact test for categorical variables, respectively. To identify clinical factors associated with early post-HT
CKD and post-HT ESKD, the association of potential risk factors with competing risk outcomes was tested using the Fine and Gray regression model. Analysis using the Generalized Estimating Equation (GEE) was applied to repeated measurements of parameters for post-HT eGFR changes. A joint model to investigate the effect of eGFR changes on survival and dialysis-free survival data was used. In the linear mixed-effects model, time and risk factors were considered as fixed effects, and time and subject were considered random effects. The survival component of the joint model consists of a Weibull model. All multivariable models were performed with variables with $P<$ 0.1 in univariable analyses. All tests were two-sided, and statistical significance was defined as $P<0.05$. Statistical analyses were conducted using SAS version 9.4 (SAS Institute Inc, Cary, NC, USA), R 3.6.1 (Vienna, Austria; htt p://www.R-project.org/), and IBM SPSS statistics 23 (IBM Corporation, Armonk, NY, USA).

Ethics approval and consent to participate: The study was approved by the Institutional Review Board of Samsung Medical Center in compliance with the Declaration of Helsinki (IRB number: 2016-06-117). The Institutional Review Board waived informed consent because data were obtained retrospectively from electronic medical records and did not contain sensitive information.

\section{Results}

Baseline characteristics: A total of 180 patients received HT from 1996 to 2015. Baseline characteristics are summarized in Table I. The median age was 50 (IQR, 38-59) years and $31.1 \%$ were female. The median baseline eGFR was 66.2 (IQR, 47.6-84.6) $\mathrm{mL} /$ minute $/ 1.73 \mathrm{~m}^{2}$ and $17.2 \%$ $(n=31)$ had preexisting CKD before HT. Among patients with pre-HT eGFR $<60 \mathrm{~mL} /$ minute $/ 1.73 \mathrm{~m}^{2}, 42.5 \%$ (31 of 73 patients) had preexisting CKD. One patient underwent simultaneous heart and kidney transplantation.

Subgroup analyses according to pre-HT eGFR and perioperative RRT application: Patients were divided into the 3 subgroups according to preoperative baseline $\mathrm{eGFR}^{16)}$ (normal and low eGFR; eGFR $\geq 50$ and $15<$ eGFR $<50 \mathrm{~mL} /$ minute $/ 1.73 \mathrm{~m}^{2}$ ) and preoperative RRT application. Baseline characteristics and post-HT outcomes according to subgroup are summarized in Table I. Patients with low baseline eGFR and preoperative RRT were more likely to have preexisting CKD compared with normal baseline eGFR (normal eGFR versus low eGFR: $7.3 \%$ versus $45.2 \%, P<0.001$; normal eGFR vs. preoperative RRT: $7.3 \%$ versus $32.0 \%, P=0.002$ ). Pre-HT albuminuria was more frequent in the preoperative RRT group compared to the normal eGFR group (normal eGFR versus preoperative RRT: $25.8 \%$ versus $68.0 \%, P<0.001$ ), but there was no difference in pre-HT albuminuria between the normal and low baseline eGFR groups. Pre-HT kidney US score did not vary between groups.

Patients with preoperative RRT were more likely to receive pre-HT mechanical support and postoperative RRT and have longer total and postoperative ICU stays compared with those with normal eGFR. One-year post-HT eGFR and CKD prevalence did not vary between the normal eGFR and preoperative RRT groups. In contrast, there 
Table I. Baseline Characteristics and Post-HT Outcomes According to Pre-HT eGFR and Preoperative RRT*

\begin{tabular}{|c|c|c|c|c|c|c|}
\hline & \multicolumn{6}{|c|}{ Pre-HT renal function group } \\
\hline & Total & $\begin{array}{l}\mathrm{eGFR} \geq 50 \\
(n=124)\end{array}$ & $\begin{array}{l}\text { eGFR } 15-50 \\
\quad(n=31)\end{array}$ & $\begin{array}{l}\text { Preoperative RRT } \\
\qquad(n=25)\end{array}$ & $P^{\dagger}$ & $P^{\ddagger}$ \\
\hline $\begin{array}{l}\text { Pre-HT eGFR } \\
\left(\mathrm{mL} / \mathrm{minute} / 1.73 \mathrm{~m}^{2}\right)\end{array}$ & $66.2(47.6-84.6)$ & $75.8(63.8-89.6)$ & $40.6(33.5-45.3)$ & NA & NA & NA \\
\hline Age (years) & $50(38-59)$ & $48(37-57)$ & $55(47-61)$ & $57(40-62)$ & 0.01 & 0.10 \\
\hline Sex (female) & $56(31.1 \%)$ & $37(29.8 \%)$ & $10(33.3 \%)$ & $9(36.0 \%)$ & 0.83 & 0.64 \\
\hline BMI $\left(\mathrm{kg} / \mathrm{m}^{2}\right)$ & $21.8(19.9-24.1)$ & $21.5(19.8,23.7)$ & $22.9(20.8,24.4)$ & $22.6(20.6,25.4)$ & 0.20 & 0.30 \\
\hline Preexisting CKD ${ }^{\S}$ & $31(17.2 \%)$ & $9(7.3 \%)$ & $14(45.2 \%)$ & $8(32.0 \%)$ & $<0.001$ & 0.002 \\
\hline $\begin{array}{l}\text { Pre-HT NT-proBNP } \\
(\mathrm{ng} / \mathrm{mL})(12)^{\#}\end{array}$ & $\begin{array}{c}5325 \\
(2550-13775)\end{array}$ & $\begin{array}{c}4536 \\
(2331-9769)\end{array}$ & $\begin{array}{c}7373 \\
(3237-13766)\end{array}$ & $\begin{array}{c}13927 \\
(5073-34211)\end{array}$ & 0.08 & 0.001 \\
\hline Pre-HT kidney US score" (5) \# & $1.27(2.56)$ & $1.32(2.12)$ & $2.21(3.32)$ & $2.00(3.37)$ & 0.44 & 0.76 \\
\hline Pre-HT albuminuria & $59(32.8 \%)$ & $32(25.8 \%)$ & $10(32.3 \%)$ & $17(68.0 \%)$ & 0.50 & $<0.001$ \\
\hline Pre-HT CCr (mL/minute) $(18)$ \# & $54.9(37.7-80.6)$ & $61.4(46.3-83.4)$ & $40.4(28.7-46.8)$ & $44.3(24.2-62.1)$ & $<0.001$ & 0.02 \\
\hline Pre-HT inotrope use & $142(78.9 \%)$ & $92(64.8 \%)$ & $27(87.1 \%)$ & $23(92.0 \%)$ & 0.16 & 0.07 \\
\hline Pre-HT ACEi/ARB use & $87(48.3 \%)$ & $53(57.3 \%)$ & $11(35.5 \%)$ & $5(20.0 \%)$ & 0.04 & 0.001 \\
\hline Pre-HT mechanical support** & $63(35.0 \%)$ & $33(26.6 \%)$ & $11(35.5 \%)$ & $18(76.0 \%)$ & 0.38 & $<0.001$ \\
\hline Pre-HT ejection fraction & $22.0(17.0-30.0)$ & $21.0(16.0-28.0)$ & $22.0(17.8-27.0)$ & $27(17.3-41.5)$ & 1.00 & 0.10 \\
\hline Postoperative RRT* & $31(17.2 \%)$ & $12(9.7 \%)$ & $6(19.4 \%)$ & $13(52.0 \%)$ & 0.21 & $<0.001$ \\
\hline Total hospital stay (days) & $66(40-100)$ & $63(38-100)$ & $62(46-79)$ & $73(60-134)$ & 0.89 & 0.05 \\
\hline Total ICU stay (days) & $12(7-25)$ & $9.5(6-18)$ & $15(8-33)$ & $27(17-45)$ & 0.05 & $<0.001$ \\
\hline Post-operative ICU stay (days) & $9(6-15)$ & $8(6-12)$ & $11(6-23)$ & $12(9-23)$ & 0.05 & $<0.001$ \\
\hline Reoperation/bleeding & $19(10.6 \%)$ & $10(8.1 \%)$ & $6(19.4 \%)$ & $3(12.0 \%)$ & 0.09 & 0.46 \\
\hline Infection & $11(6.1 \%)$ & $28(4.0 \%)$ & $14(6.5 \%)$ & $4(16.0 \%)$ & 0.63 & 0.04 \\
\hline Post-transplant 1-year eGFR & $66.9(22.6)$ & $72.0(21.8)$ & $50.5(17.0)$ & $60.5(22.8)$ & $<0.001$ & 0.04 \\
\hline Post-transplant CKD at 1-year & $58(32.2 \%)$ & $32(30.5 \%)$ & $16(64.0 \%)$ & $10(52.6 \%)$ & 0.003 & 0.07 \\
\hline Death within 1 year & $30(16.7 \%)$ & $19(15.3 \%)$ & $5(16.1 \%)$ & $6(24.0 \%)$ & 1.00 & 0.38 \\
\hline Death within 6 months & $18(10.0 \%)$ & $10(8.1 \%)$ & $3(9.7 \%)$ & $5(20.0 \%)$ & 0.72 & 0.14 \\
\hline
\end{tabular}

Continuous variables are expressed as the mean (standard deviation) or median (interquartile range), as appropriate, and categorical variables are expressed as number (percentage). Statistical significance of this table was defined as $P<0.025$ according to the Bonferroni correction for multiple comparisons. ACEi/ARB indicates angiotensin converting enzyme inhibitor/angiotensin II receptor blocker; BMI, body mass index; CCr, creatinine clearance; CKD, chronic kidney disease; eGFR, estimated glomerular filtration rate; HT, heart transplantation; ICU, intensive care unit; NA, not applicable; NT-proBNP, N-terminal prohormone of brain natriuretic peptide; RRT, renal replacement therapy; and US, ultrasound. *Pre and postoperative RRT: patients who received RRT within 1 month before and after HT, respectively. ${ }^{\dagger} P$ : eGFR $\geq 50$ versus eGFR $15-50$. ${ }^{\ddagger} P$ : eGFR $\geq 50$ versus Preoperative RRT. ${ }^{\S} \mathrm{CKD}$ : defined as eGFR less than $60 \mathrm{~mL} /$ minute $/ 1.73 \mathrm{~m}^{2}$ for at least 3 months before HT. "Kidney US score: sum of scores was calculated - normal (0), increased echogenicity (1), loss of corticomedullary differentiation (2), cortical thinning (3), and smallsized kidney (4). \#The number of missing values. **Pre-HT mechanical support included intra-aortic ballooning pump, extracorporeal membrane oxygenation, and ventricular assist device.

were no differences in pre-HT mechanical support, postoperative RRT and ICU stay between the normal and low eGFR groups, but 1-year post-HT eGFR was lower (normal eGFR vs. low eGFR: $72.0 \pm 21.8$ versus $50.1 \pm 17.0$ $\mathrm{mL} /$ minute $\left./ 1.73 \mathrm{~m}^{2}, P<0.001\right)$ and 1 -year post-HT CKD prevalence was higher (normal eGFR versus low eGFR: $30.5 \%$ versus $64.0 \%, P=0.003$ ) in the low eGFR group compared with the normal eGFR group. There was no difference in mortality within 1 year between groups.

Changes in eGFR up to 3 years post-HT depending on preoperative RRT: We further analyzed serial changes in eGFR from the operative day to 3 years depending on preoperative RRT (Figure). eGFR abruptly increased during the first month postoperative, decreased during the third to sixth months, and then was maintained stably for up to 3 years. There were no significant differences in renal function between the two groups at 1 month post-HT $(P=0.57)$ and up to 3 years post-HT $(P=0.88)$.

Factors associated with early post-HT CKD and postHT ESKD: Early post-HT CKD (developing within 1 year postoperative) and post-HT ESKD developed in 61 $(33.9 \%)$ and $8(4.4 \%)$ of 180 patients, respectively. There were no subsequent kidney transplantations after HT up to the end of follow-up. As shown in Table II, old age was independently associated with early post-HT CKD (hazard ratio [HR] 1.04, 95\% confidence interval [CI] 1.02-1.06, $P<0.001)$. Preexisting $\mathrm{CKD}$ and albuminuria tended to increase the risk of early post-HT CKD (preexisting CKD: HR $1.70, P=0.10$; albuminuria: HR $1.59, P=0.08$ ). The mean tacrolimus level was lower in patients with early post-HT CKD compared to those without early post-HT CKD, but there was no difference in the mean trough level of tacrolimus (Supplemental Table I). Mean cyclosporine dose and trough level were similar between patients with and without early post-HT CKD. The proportion of patients prescribed with mycophenolate was smaller in patients with early post-HT CKD than in those without early post-HT CKD $(71.4 \%$ versus $88.2 \%, P=$ 0.012). Immunosuppressants and rejection episodes were not associated with early post-HT CKD (Supplemental Table II).

For post-HT ESKD, old age and preexisting CKD were independently associated with post-HT ESKD (age: HR 1.07, $P=0.03$; preexisting CKD: HR 5.61, $P<$ 0.001) (Table III). Pre-HT renal function categories, including preoperative RRT, were not associated with either 


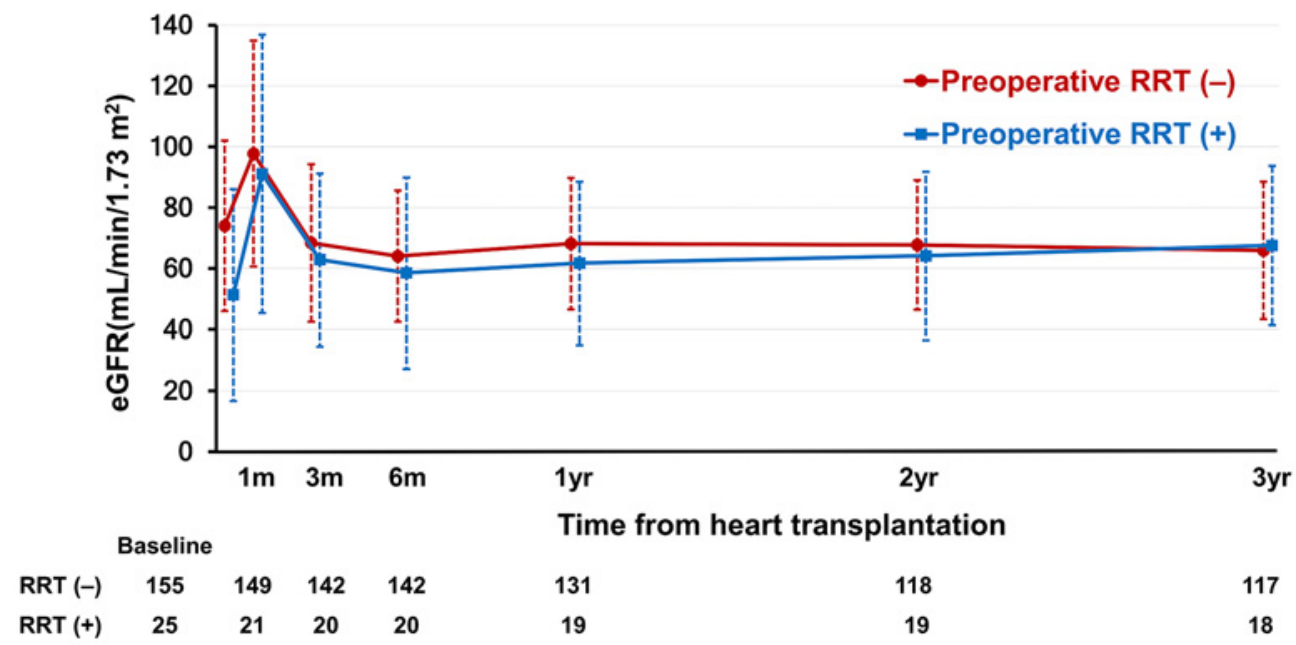

Figure. Serial changes of eGFR up to 3 years post-HT according to preoperative RRT. There were no significant differences in post-HT eGFR according to preoperative RRT. Error bars represent standard deviation. The number of patients at risk is presented at the bottom of the figure. eGFR indicates estimated glomerular filtration rate; HT, heart transplantation; and RRT, renal replacement therapy.

Table II. Predictive Factors of Early Post-HT CKD (Within 1 Year After HT)

\begin{tabular}{|c|c|c|c|c|c|c|}
\hline & \multicolumn{3}{|c|}{ Univariable } & \multicolumn{3}{|c|}{ Multivariable } \\
\hline & HR & $95 \% \mathrm{CI}$ & $P$ & HR & $95 \% \mathrm{CI}$ & $P$ \\
\hline Pre-HT renal function categories & & & 0.003 & & & 0.26 \\
\hline eGFR $\geq 50\left(\mathrm{~mL} /\right.$ minute $\left./ 1.73 \mathrm{~m}^{2}\right)$ & & & Ref & & & Ref \\
\hline $15<\mathrm{eGFR}<50\left(\mathrm{~mL} /\right.$ minute $\left./ 1.73 \mathrm{~m}^{2}\right)$ & 2.60 & $1.50-4.49$ & 0.007 & 1.65 & $0.84-3.22$ & 0.15 \\
\hline Preoperative RRT* & 1.60 & $0.84-3.06$ & 0.15 & 0.98 & $0.49-1.97$ & 0.96 \\
\hline Age (years) & 1.05 & $1.03-1.07$ & $<0.001$ & 1.04 & $1.02-1.06$ & $<0.001$ \\
\hline Sex (female) & 0.95 & $0.06-1.60$ & 0.85 & & & \\
\hline BMI $\left(\mathrm{kg} / \mathrm{m}^{2}\right)$ & 1.02 & $0.97-1.08$ & 0.49 & & & \\
\hline Preexisting $\mathrm{CKD}^{\dagger}$ & 2.83 & $1.69-4.73$ & $<0.001$ & 1.70 & $0.91-3.16$ & 0.10 \\
\hline Kidney US score & 1.03 & $0.95-1.12$ & 0.44 & & & \\
\hline NT-proBNP (ng/mL) & 1.13 & $0.66-1.92$ & 0.66 & & & \\
\hline Albuminuria & 1.56 & $0.96-2.52$ & 0.07 & 1.59 & $0.94-2.58$ & 0.08 \\
\hline Pre-HT mechanical support ${ }^{\S}$ & 1.46 & $0.91-2.35$ & 0.12 & & & \\
\hline Pre-HT ejection fraction $(\%)$ & 1.00 & $0.98-1.02$ & 0.95 & & & \\
\hline
\end{tabular}

Multivariable analysis was performed using the variables $P<0.1$ in univariable analyses. NTproBNP was non-normally distributed and was therefore transformed using log transformation prior to its inclusion in statistical analysis. BMI indicates body mass index; CI, confidence interval; CKD, chronic kidney disease; eGFR, estimated glomerular filtration rate; HT, heart transplantation; HR, hazard ratio; NT-proBNP, N-terminal prohormone of brain natriuretic peptide; Ref, reference; RRT, renal replacement therapy; and US, ultrasound. *Preoperative RRT group: patients who received RRT within 1 month before HT. ${ }^{+}$Preexisting CKD: defined as eGFR less than $60 \mathrm{~mL} / \mathrm{minute} / 1.73 \mathrm{~m}^{2}$ for at least 3 months before HT. ${ }^{*}$ Kidney US score: sum of scores was calculated - normal (0), increased echogenicity (1), loss of corticomedullary differentiation (2), cortical thinning (3), and small-sized kidney (4). \$Pre-HT mechanical support included intra-aortic ballooning pump, extracorporeal membrane oxygenation, and ventricular assist device.

early post-HT CKD or post-HT ESKD in multivariable analysis.

Factors associated with post-HT eGFR: Post-HT eGFR was lower 6 to 9 months post-HT compared with 3 months post-HT, but there was no difference between 3month post-HT eGFR and 1- to 3-year post-HT eGFR. In multivariable analysis, preoperative low eGFR, old age, and preexisting CKD were independently associated with low post-HT eGFR (low eGFR: $\beta-10.07, P=0.005$; old age: $\beta-0.66, P<0.001$; preexisting CKD: $\beta-14.03, P<$ 0.001) (Table IV). Preoperative RRT was not associated with low post-HT eGFR. Further analysis of post-HT
eGFR, including the immunosuppressant administration status and rejection after HT, showed that the use of mycophenolate was associated with higher post-HT eGFR (Supplemental Table III).

Factors associated with significant improvements in renal function: Changes in renal function were expressed as $\% \Delta \mathrm{eGFR}$, and a higher $\% \Delta \mathrm{eGFR}$ signified greater renal function improvements after HT. Young age, lower pre-HT eGFR, and higher 1-month post-HT \% 1 eGFR were associated with significant improvements in renal function $(\% \Delta \mathrm{eGFR}>15 \%)$ in multivariable analysis (age: HR 0.93, $P=0.001$; pre-HT eGFR: HR 0.94, $P=0.001$; 
Table III. Predictive Factors of Post-HT End-Stage Kidney Disease

\begin{tabular}{|c|c|c|c|c|c|c|}
\hline & \multicolumn{3}{|c|}{ Univariable } & \multicolumn{3}{|c|}{ Multivariable } \\
\hline & HR & $95 \% \mathrm{CI}$ & $P$ & $\mathrm{HR}$ & $95 \% \mathrm{CI}$ & $P$ \\
\hline Pre-HT renal function categories & & & 0.09 & & & 0.70 \\
\hline eGFR $\geq 50\left(\mathrm{~mL} /\right.$ minute $\left./ 1.73 \mathrm{~m}^{2}\right)$ & Ref & & & Ref & & \\
\hline $15<\mathrm{eGFR}<50\left(\mathrm{~mL} /\right.$ minute $\left./ 1.73 \mathrm{~m}^{2}\right)$ & 4.94 & $1.15-21.29$ & 0.03 & 1.63 & $0.51-5.17$ & 0.41 \\
\hline Preoperative RRT* & 2.85 & $9.34-22.70$ & 0.33 & 0.97 & $0.20-4.83$ & 0.98 \\
\hline Age (years) & 1.08 & $1.03-1.125$ & $<0.001$ & 1.07 & $1.01-1.14$ & 0.03 \\
\hline Sex (female) & 2.01 & $0.53-7.56$ & 0.30 & & & \\
\hline $\operatorname{BMI}\left(\mathrm{kg} / \mathrm{m}^{2}\right)$ & 0.98 & $0.84-1.14$ & 0.77 & & & \\
\hline Preexisting $\mathrm{CKD}^{\dagger}$ & 10.32 & $2.80-38.04$ & $<0.001$ & 5.61 & $2.19-14.33$ & $<0.001$ \\
\hline Kidney US score ${ }^{\ddagger}$ & 1.10 & $0.86-1.41$ & 0.46 & & & \\
\hline NT-proBNP (ng/mL) & 1.04 & $0.36-2.98$ & 0.94 & & & \\
\hline Albuminuria & 1.33 & $0.28-6.36$ & 0.72 & & & \\
\hline Pre-HT mechanical support ${ }^{\S}$ & 0.33 & $0.04-2.65$ & 0.30 & & & \\
\hline Pre-HT ejection fraction $(\%)$ & 0.97 & $0.92-1.03$ & 0.35 & & & \\
\hline
\end{tabular}

Multivariate analysis was performed using the variables $P<0.1$ in univariate analyses. NTproBNP was non-normally distributed and was therefore transformed using $\log$ transformation prior to its inclusion in statistical analysis. BMI indicates body mass index; CI, confidence interval; CKD, chronic kidney disease; eGFR, estimated glomerular filtration rate; HT, heart transplantation; HR, hazard ratio; NT-proBNP, N-terminal prohormone of brain natriuretic peptide; RRT, renal replacement therapy; and US, ultrasound. *Preoperative RRT group: patients who received RRT within 1 month before HT. "Preexisting CKD: defined as eGFR less than $60 \mathrm{~mL} /$ minute $/ 1.73 \mathrm{~m}^{2}$ for at least 3 months. "Kidney US score: sum of scores was calculated - normal (0), increased echogenicity (1), loss of corticomedullary differentiation (2), cortical thinning (3), and small-sized kidney (4). \$Pre-HT mechanical support included intra-aortic ballooning pump, extracorporeal membrane oxygenation, and ventricular assist device.

Table IV. Predictive Factors of Post-HT eGFR

\begin{tabular}{|c|c|c|c|c|c|c|}
\hline & \multicolumn{3}{|c|}{ Univariable } & \multicolumn{3}{|c|}{ Multivariable } \\
\hline & $\beta$ & SE & $P$ & $\beta$ & SE & $P$ \\
\hline Time & & & 0.007 & & & 0.01 \\
\hline 3 months & & & Ref & & & Ref \\
\hline 6 months & -4.22 & 1.51 & 0.005 & -4.14 & 1.52 & 0.007 \\
\hline 9 months & -5.09 & 2.23 & 0.02 & -4.18 & 1.97 & 0.03 \\
\hline 1 year & -0.53 & 1.88 & 0.78 & -0.75 & 1.82 & 0.68 \\
\hline 2 years & -0.40 & 1.89 & 0.83 & -0.92 & 1.80 & 0.61 \\
\hline 3 years & -0.12 & 2.08 & 0.95 & -0.42 & 1.97 & 0.83 \\
\hline Time \& pre-HT renal function categories & & & 0.71 & & & \\
\hline Pre-HT renal function category & & & $<0.001$ & & & 0.02 \\
\hline $\mathrm{eGFR} \geq 50\left(\mathrm{~mL} /\right.$ minute $\left./ 1.73 \mathrm{~m}^{2}\right)$ & & & Ref & & & Ref \\
\hline $15<$ eGFR $<50\left(\mathrm{~mL} /\right.$ minute $\left./ 1.73 \mathrm{~m}^{2}\right)$ & -20.78 & 3.61 & $<0.001$ & -10.07 & 3.61 & 0.005 \\
\hline Preoperative RRT* & -9.66 & 5.02 & 0.05 & -4.19 & 4.06 & 0.30 \\
\hline Age (years) & -0.84 & 0.11 & $<0.001$ & -0.66 & 0.10 & $<0.001$ \\
\hline Sex (female) & -3.07 & 3.64 & 0.40 & & & \\
\hline BMI $\left(\mathrm{kg} / \mathrm{m}^{2}\right)$ & 0.44 & 0.57 & 0.43 & & & \\
\hline Preexisting $\mathrm{CKD}^{\dagger}$ & -26.39 & 3.09 & $<0.001$ & -14.03 & 3.64 & $<0.001$ \\
\hline Kidney US score & -1.04 & 0.52 & 0.05 & -0.53 & 0.37 & 0.15 \\
\hline NT-proBNP (ng/mL) & -4.44 & 3.30 & 0.18 & & & \\
\hline Albuminuria & -2.77 & 3.51 & 0.43 & & & \\
\hline Pre-HT mechanical support ${ }^{\S}$ & -0.59 & 3.53 & 0.87 & & & \\
\hline Pre-HT ejection fraction $(\%)$ & 0.08 & 0.12 & 0.47 & & & \\
\hline
\end{tabular}

Multivariable analysis was performed using the variables $P<0.1$ in univariable analysis. NTproBNP was non-normally distributed and was therefore transformed using log transformation prior to its inclusion in statistical analysis. BMI indicates body mass index; $\beta, \beta$ coefficient; CKD, chronic kidney disease; eGFR, estimated glomerular filtration rate; HT, heart transplantation; NT-proBNP, N-terminal prohormone of brain natriuretic peptide; Ref, reference; RRT, renal replacement therapy; SE, standard error; and US, ultrasound. *Preoperative RRT group: patients who received RRT within 1 month before HT. 'Preexisting CKD: defined as eGFR less than $60 \mathrm{~mL} /$ minute $/ 1.73 \mathrm{~m}^{2}$ for at least 3 months before HT. ${ }^{*}$ Kidney US score: sum of scores was calculated - normal (0), increased echogenicity (1), loss of corticomedullary differentiation (2), cortical thinning (3), and small-sized kidney (4). \$Pre-HT mechanical support included intra-aortic balloon pump, extracorporeal membrane oxygenation, and ventricular assist device. 
Table V. Predictive Factors of Significant Improvement of Renal Function $(\% \Delta \mathrm{eGFR} *>15 \%)$

\begin{tabular}{lcccccc}
\hline & \multicolumn{3}{c}{ Univariable } & \multicolumn{3}{c}{ Multivariable } \\
& OR & $95 \%$ CI & $P$ & OR & $95 \%$ CI & $P$ \\
\hline Age (years) & 0.98 & $0.96-1.00$ & 0.08 & 0.93 & $0.89-0.97$ & 0.001 \\
Sex (female) & 0.97 & $0.47-2.03$ & 0.94 & & & \\
BMI (kg/m²) & 1.02 & $0.94-1.11$ & 0.63 & & & \\
Pre-HT uric acid (mg/dL) & 1.12 & $1.00-1.24$ & 0.03 & 1.00 & $0.86-1.17$ & 0.99 \\
Pre-HT NT-proBNP & 1.34 & $0.98-1.82$ & 0.07 & 1.01 & $0.61-1.66$ & 0.97 \\
Preexisting CKD & 1.84 & $0.72-4.68$ & 0.20 & & & \\
Pre-HT albuminuria & 1.31 & $0.65-2.55$ & 0.45 & & & \\
Pre-HT kidney US score ${ }^{\ddagger}$ & 0.95 & $0.83-1.09$ & 0.47 & & & \\
Perioperative RRT ${ }^{\S}$ & 4.70 & $1.91-11.53$ & 0.001 & 2.73 & $0.68-10.89$ & 0.16 \\
Pre-HT mechanical support" & 1.49 & $0.74-3.01$ & 0.27 & & & \\
Pre-HT eGFR & 0.94 & $0.92-0.96$ & $<0.001$ & 0.94 & $0.91-0.97$ & 0.001 \\
CCr- eGFR & 1.01 & $1.00-1.02$ & 0.04 & 0.99 & $0.98-1.01$ & 0.37 \\
\% $\Delta$ eGFR 1 week post-HT & 1.02 & $1.01-1.03$ & $<0.001$ & 1.01 & $1.00-1.03$ & 0.15 \\
\% $\Delta$ eGFR 1 month post-HT & 1.02 & $1.01-1.03$ & $<0.001$ & 1.01 & $1.00-1.02$ & 0.03 \\
\hline
\end{tabular}

Multivariable analysis was performed using the variables $P<0.1$ in univariable analyses. NTproBNP was non-normally distributed and was therefore transformed using log transformation prior to its inclusion in statistical analysis. BMI indicates body mass index; $\mathrm{CCr}$, creatinine clearance; $\mathrm{CI}$, confidence interval; CKD, chronic kidney disease; eGFR, estimated glomerular filtration rate; HT, heart transplantation; NT-proBNP, N-terminal prohormone of brain natriuretic peptide; OR, odds ratio; RRT, renal replacement therapy; and US, ultrasound. *\% $\%$ eGFR: $100 \times[$ (post-HT eGFR) $-($ pre-HT eGFR) $] /$ pre-HT eGFR. ${ }^{\dagger} \mathrm{CKD}$ : defined as eGFR less than $60 \mathrm{~mL} /$ minute $/ 1.73 \mathrm{~m}^{2}$ for at least 3 months before HT. ${ }^{*}$ Kidney US score: sum of scores was calculated - normal (0), increased echogenicity (1), loss of corticomedullary differentiation (2), cortical thinning (3), and small-sized kidney (4). \$Perioperative RRT group: patients who received RRT within 1 month before or after HT. "Pre-HT mechanical support included intra-aortic balloon pump, extracorporeal membrane oxygenation, and ventricular assist device.

Table VI. Predictive Factors of Overall Survival and Chronic Dialysis-Free Survival from the Joint Model Adjusting for the Effect of Post-HT eGFR Changes

\begin{tabular}{|c|c|c|c|c|c|c|}
\hline & \multicolumn{3}{|c|}{ Overall survival } & \multicolumn{3}{|c|}{ Chronic dialysis-free survival } \\
\hline & Adjusted $\beta$ & SE & $P$ & Unadjusted $\beta$ & SE & $P$ \\
\hline \multicolumn{7}{|l|}{ Pre-HT renal function categories } \\
\hline $\mathrm{eGFR} \geq 50\left(\mathrm{~mL} /\right.$ minute $\left./ 1.73 \mathrm{~m}^{2}\right)$ & & & Ref & & & Ref \\
\hline $15<\mathrm{eGFR}<50\left(\mathrm{~mL} /\right.$ minute $\left./ 1.73 \mathrm{~m}^{2}\right)$ & 0.53 & 0.45 & 0.24 & 2.23 & 1.46 & 0.12 \\
\hline Preoperative RRT* & -0.44 & 0.52 & 0.40 & 1.67 & 1.47 & 0.26 \\
\hline Age (years) & 0.02 & 0.02 & 0.14 & 0.08 & 0.05 & 0.12 \\
\hline Sex (female) & & & & 1.26 & 1.20 & 0.29 \\
\hline BMI $\left(\mathrm{kg} / \mathrm{m}^{2}\right)$ & & & & 0.18 & 0.19 & 0.35 \\
\hline Preexisting $\mathrm{CKD}^{\dagger}$ & 0.59 & 0.46 & 0.20 & 3.28 & 3.03 & 0.28 \\
\hline Kidney US score & -0.03 & 0.06 & 0.60 & 0.02 & 0.16 & 0.93 \\
\hline NT-proBNP (ng/mL) & -0.19 & 0.29 & 0.50 & 0.39 & 0.79 & 0.62 \\
\hline Albuminuria & & & & 0.36 & 1.04 & 0.73 \\
\hline Pre-HT mechanical support ${ }^{\S}$ & 0.73 & 0.32 & 0.02 & -1.84 & 1.71 & 0.28 \\
\hline Pre-HT ejection fraction $(\%)$ & & & & 0.06 & 0.07 & 0.41 \\
\hline
\end{tabular}

Multivariable analysis was performed using the variables $P<0.1$ in univariable analyses. NTproBNP was non-normally distributed and was therefore transformed using log transformation prior to its inclusion in statistical analysis. BMI indicates body mass index; $\beta, \beta$ coefficient; CKD, chronic kidney disease; eGFR, estimated glomerular filtration rate; HT, heart transplantation; NT-proBNP, N-terminal prohormone of brain natriuretic peptide; Ref, reference; RRT, renal replacement therapy; SE, standard error; and US, ultrasound. *Preoperative RRT group: patients who received RRT within 1 month before HT. "Preexisting CKD: defined as eGFR less than $60 \mathrm{~mL} /$ minute $/ 1.73 \mathrm{~m}^{2}$ for at least 3 months before HT. ${ }^{*}$ Kidney US score: sum of scores was calculated - normal (0), increased echogenicity (1), loss of corticomedullary differentiation (2), cortical thinning (3), and small-sized kidney (4). ${ }^{\S}$ Pre-HT mechanical support included intra-aortic balloon pump, extracorporeal membrane oxygenation, and ventricular assist device.

$\% \Delta \mathrm{eGFR} 1$ month post-HT: HR 1.01, $P=0.03$ ) (Table $\mathrm{V})$.

Factors associated with overall survival and chronic dialysis-free survival: We conducted further analyses using a joint model to investigate factors associated with overall and chronic dialysis-free survival, considering and adjusting for the effects of eGFR changes on death (Table VI). In multivariable analysis of the joint model, preoperative mechanical support was associated with overall survival (adjusted $\beta=0.73, P=0.02$ ). Preoperative RRT and 
preexisting CKD were not associated with overall survival. There were no significant variables associated with chronic dialysis-free survival in univariable analyses. Multivariable analysis for chronic dialysis-free survival was not statistically feasible in the joint model as ESKD events were rare.

\section{Discussion}

Preoperative factors affecting renal function after HT were evaluated in 180 patients receiving HT. Preexisting CKD was associated with post-HT ESKD and post-HT eGFR and was a more relevant risk factor for post-HT renal outcome compared with preoperative eGFR per se. Age was associated with early post-HT CKD. However, preoperative renal function, including preoperative RRT, was not associated with post-HT mortality. Significant improvement in renal function after HT was associated with young age, lower pre-HT eGFR, and a greater degree of renal function improvement within 1 month after HT.

Preexisting CKD was independently associated with post-HT ESKD and post-HT eGFR and tended to be associated with early post-HT CKD. On the other hand, low pre-HT eGFR and preoperative RRT were not associated with early post-HT CKD or post-HT ESKD. Old age, female sex, and DM were relatively consistent factors associated with worse renal function after HT across previous studies. ${ }^{717-21)}$ Although one study showed that preoperative low GFR was associated with post-HT ESKD,${ }^{16}$ ) more recent studies have indicated that preoperative renal function is not associated with post-HT advanced CKD or ESKD, similar to our findings. ${ }^{19,21)}$ Preexisting CKD has not been evaluated as a factor affecting renal outcome in previous studies. Our results identified preexisting CKD as a clinically more relevant and reliable factor associated with post-HT renal outcome than pre-HT eGFR per se as well as preoperative RRT. This is probably because low preoperative GFR before HT includes reversible AKI caused by cardiorenal syndrome. Therefore, a substantial portion of these patients may show renal functional improvement after HT. Only half of patients with pre-HT eGFR $<60 \mathrm{~mL} /$ minute $/ 1.73 \mathrm{~m}^{2}$ had preexisting $\mathrm{CKD}$, implying that half of them had AKI. Our findings support that AKI due to cardiorenal syndrome can be significantly improved after HT.

Except for one recent study with a relatively small number of patients and a short-term follow-up period, no previous studies focused on factors associated with improvement of renal function after $\mathrm{HT}^{22)}$ Given that patients with severe renal dysfunction should be considered for HKT, predicting the possibility of renal function improvement after HT is an important issue. Our results suggest that kidney function may improve even in patients with low pre-HT eGFR, especially in young patients. In addition, renal function changes within the first month after HT were significantly associated with renal function changes during the first year after HT, suggesting the importance of renoprotective strategies for improving renal function during the early postoperative period after HT.

We demonstrated that preoperative RRT was not associated with mortality, which was analyzed using either simple 1-year mortality or multivariable analysis using a joint model. Patients receiving dialysis before HT were reported to show poor outcomes after HT alone and HKT was reported to improve survival in these patients. ${ }^{23-25)}$ However, it remains unclear whether these patients had dialysis-dependent ESKD or temporary dialysis-required AKI. No previous study evaluated the impact of AKI requiring preoperative RRT on post-HT mortality or renal outcome. Considering our results, AKI requiring RRT before HT should not be regarded as a contraindication to HT alone unless ESKD is definitively diagnosed.

Low pre-HT eGFR was not associated with high mortality or post-HT poor renal outcome. However, there were only 3 patients who had eGFR $<30 \mathrm{~mL} /$ minute/1.73 $\mathrm{m}^{2}$ and did not receive preoperative RRT, so the risk of death in patients with eGFR $<30 \mathrm{~mL} /$ minute $/ 1.73 \mathrm{~m}^{2}$ remains inconclusive. Previous studies have shown that, although not in some studies, pre-HT renal dysfunction has worse long-term survival after HT alone. ${ }^{8,21,23)}$ Although it is not apparent why previous results varied, eGFR by serum creatinine-based formulas in HT recipients has been reported to be a poor representation of actual GFR. ${ }^{26-28)}$ Kolsrud, et al. measured GFR directly using ${ }^{15} \mathrm{Cr}$-EDTA or iohexol before and after HT and showed that pre-HT GFR was not associated with post-HT survival, which agrees with our data. ${ }^{21)}$

Some limitations to our study are worth mentioning. First, the study design was a single-center retrospective cohort study raising generalization issues. Although we performed multivariable analyses using a variety of clinical factors, our results may still be affected by unmeasured confounding factors. Second, the study population was relatively small, so this study may have low power to identify small but significant factors. However, various variables that reflect not only kidney status but also overall patient medical condition, such as kidney US score and preoperative mechanical support including extracorporeal membrane oxygenation were included to improve clinical relevance. Third, direct measurement of GFR using ${ }^{15} \mathrm{Cr}$-EDTA or iohexol was not included in our analyses because of the retrospective study design. Fourth, the follow-up period was relatively short. However, all patients were followed for at least 3 years post-HT or death and there were no significant changes in renal function from 1 to 3 years after HT in our study, indicating that renal function at 1 to 3 years can reasonably reflect renal outcomes.

\section{Conclusions}

These data suggest that timely HT before CKD development may improve renal outcome even in HF patients with severe AKI, especially young age patients, and pre-HT AKI may not be a contraindication to HT alone even if RRT is required. Furthermore, close monitoring of renal function and careful renoprotective management during the early postoperative period, especially within the first month after HT, may be critical for improving postHT renal outcome. 


\section{Acknowledgment}

We would like to express our appreciation to the staff of the organ transplantation center and the nursing team of the cardiac surgical ICU at Samsung Medical Center, Sungkyunkwan University School of Medicine.

\section{Disclosure}

Conflicts of interest: The authors have no conflicts of interest to declare.

\section{References}

1. Braam B, Joles JA, Danishwar AH, Gaillard CA. Cardiorenal syndrome--current understanding and future perspectives. Nat Rev Nephrol 2014; 10: 48-55.

2. McAlister FA, Ezekowitz J, Tonelli M, Armstrong PW. Renal insufficiency and heart failure: prognostic and therapeutic implications from a prospective cohort study. Circulation 2004; 109: 1004-9.

3. Smith GL, Lichtman JH, Bracken MB, et al. Renal impairment and outcomes in heart failure: systematic review and metaanalysis. J Am Coll Cardiol 2006; 47: 1987-96.

4. Dellgren G, Geiran O, Lemstrom K, et al. Three decades of heart transplantation in Scandinavia: long-term follow-up. Eur J Heart Fail 2013; 15: 308-15.

5. Dellgren G, Westerlind A, Liden H, et al. Continuous improvement in outcome after heart transplantation - Long-term followup after three decades of experience. Int J Cardiol 2017; 231: 188-94.

6. Odim J, Wheat J, Laks H, et al. Peri-operative renal function and outcome after orthotopic heart transplantation. J Heart Lung Transplant 2006; 25: 162-6.

7. Ojo AO, Held PJ, Port FK, et al. Chronic renal failure after transplantation of a nonrenal organ. N Engl J Med 2003; 349: 931-40.

8. Habib PJ, Patel PC, Hodge D, et al. Pre-orthotopic heart transplant estimated glomerular filtration rate predicts post-transplant mortality and renal outcomes: An analysis of the UNOS database. J Heart Lung Transplant 2016; 35: 1471-9.

9. Ostermann ME, Rogers CA, Saeed I, Nelson SR, Murday AJ. Pre-existing renal failure doubles 30-day mortality after heart transplantation. J Heart Lung Transplant 2004; 23: 1231-7.

10. Mehra MR, Canter CE, Hannan MM, et al. The 2016 International Society for Heart Lung Transplantation listing criteria for heart transplantation: A 10-year update. J Heart Lung Transplant 2016; 35: 1-23.

11. Goupil R, Bonnardeaux A, Boucher A, et al. Difficulty of patient selection in a combined heart-kidney transplant: a case report. Exp Clin Transplant 2014; 12: 273-6.

12. Levey AS, Coresh J, Greene T, et al. Using standardized serum creatinine values in the modification of diet in renal disease study equation for estimating glomerular filtration rate. Ann Intern Med 2006; 145: 247-54.
13. Yaprak M, Çakır Ö, Turan MN, et al. Role of ultrasonographic chronic kidney disease score in the assessment of chronic kidney disease. Int Urol Nephrol 2017; 49: 123-31.

14. Kidney Disease: Improving Global Outcomes (KDIGO) CKD Work Group. KDIGO clinical practice guideline for the evaluation and management of chronic kidney disease. Kidney Int Suppl 2013; 3: 1-150.

15. Arora S, Andreassen A, Simonsen S, et al. Prognostic importance of renal function 1 year after heart transplantation for allcause and cardiac mortality and development of allograft vasculopathy. Transplantation 2007; 84: 149-54.

16. Rubel JR, Milford EL, McKay DB, Jarcho JA. Renal insufficiency and end-stage renal disease in the heart transplant population. J Heart Lung Transplant 2004; 23: 289-300.

17. Bloom RD, Reese PP. Chronic kidney disease after nonrenal solid-organ transplantation. J Am Soc Nephrol 2007; 18: 303141

18. Gonzalez-Vilchez F, Arizon JM, Segovia J, et al. Chronic renal dysfunction in maintenance heart transplant patients: the ICEBERG study. Transplant Proc 2014; 46: 14-20.

19. Hamour IM, Omar F, Lyster HS, Palmer A, Banner NR. Chronic kidney disease after heart transplantation. Nephrol Dial Transplant 2009; 24: 1655-62.

20. Guitard J, Ribes D, Kamar N, et al. Predictive factors for chronic renal failure one year after orthotopic liver transplantation. Ren Fail 2006; 28: 419-25.

21. Kolsrud O, Karason K, Holmberg E, et al. Renal function and outcome after heart transplantation. J Thorac Cardiovasc Surg 2018; 155: 1593-604.e1.

22. Ivey-Miranda JB, Farrero-Torres M, Flores-Umanzor E, Santiago E, Perez-Villa F. Predictors of Improvement in Renal Function After Heart Transplant. Exp Clin Transplant 2019; 17: 387-92.

23. Schaffer JM, Chiu P, Singh SK, Oyer PE, Reitz BA, Mallidi HR. Heart and combined heart-kidney transplantation in patients with concomitant renal insufficiency and end-stage heart failure. Am J Transplant 2014; 14: 384-96.

24. Gill J, Shah T, Hristea I, et al. Outcomes of simultaneous heartkidney transplant in the US: a retrospective analysis using OPTN/UNOS data. Am J Transplant 2009; 9: 844-52.

25. Kilic A, Grimm JC, Whitman GJ, et al. The survival benefit of simultaneous heart-kidney transplantation extends beyond dialysis-dependent patients. Ann Thorac Surg 2015; 99: 1321-7.

26. Delanaye P, Nellessen E, Grosch S, et al. Creatinine-based formulae for the estimation of glomerular filtration rate in heart transplant recipients. Clin Transplant 2006; 20: 596-603.

27. Kolsrud O, Ricksten SE, Holmberg E, et al. Measured and not estimated glomerular filtration rate should be used to assess renal function in heart transplant recipients. Nephrol Dial Transplant 2016; 31: 1182-9.

28. Shaffi K, Uhlig K, Perrone RD, et al. Performance of creatininebased GFR estimating equations in solid-organ transplant recipients. Am J Kidney Dis 2014; 63: 1007-18.

\section{Supplemental Files}

Supplemental Tables I-III

Please see supplemental files; https://doi.org/10.1536/ihj.20-775 